\title{
Guamanian or Chamorro
}

National Cancer Institute

\section{Source}

National Cancer Institute. Guamanian or Chamorro. NCI Thesaurus. Code C43837.

A person having origins in any of the original peoples of Guam or the Mariana Islands. 